\title{
Dispositional Essentialism and the Nature of Powerful Properties
}

\author{
William A. Bauer \\ North Carolina State University \\ BIBLID [0873-626X (2013) 35; pp. 1-19]
}

DOI: $10.2478 /$ disp-2013-0001

\begin{abstract}
Dispositional essentialism maintains that all sparse properties are essentially powerful. Two conceptions of sparse properties appear compatible with dispositional essentialism: sparse properties as pure powers or as powerful qualities. This paper compares the two views, criticizes the powerful qualities view, and then develops a new theory of pure powers, termed Point Theory. This theory neutralizes the main advantage powerful qualities appear to possess over pure powers - explaining the existence of powers during latency periods. The paper discusses the relation between powers and space-time points, whether pure powers need to occupy space, and how to account for the movement of pure powers through space-time. Given Point Theory, dispositional essentialists should maintain that sparse properties are pure powers.
\end{abstract}

\section{Keywords}

Dispositions, Powers, Powerful Qualities, Essentialism, Sparse Properties.

\section{Two theories of powerful sparse properties}

Dispositional essentialism maintains that all sparse properties are essentially dispositional or powerful. ${ }^{1}$ Sparse properties are the natural properties, including at least the fundamental properties, as Lewis (1986b: 59-61) conceives them. According to dispositional essentialism, every sparse property has its power (or powers) essentially; two or more sparse properties are distinguished by their powers. On this

\footnotetext{
${ }^{1}$ I use the terms 'powerful' and 'dispositional', and 'power' and 'disposition', interchangeably.

Disputatio, Vol. V, No. 35, May 2013
}

Received: 08/08/2012. Revised: 23/12/2012. Accepted: 30/01/2013 
view, defended most prominently by Bird 2007 and Mumford 2004, sparse properties are non-categorical. If sparse properties are categorical as Armstrong 2004 and Lewis 1986a maintain, then their powers are non-essential and vary in accordance with the laws of nature operating at a given world.

Dispositional essentialism appears to require that sparse properties are pure powers. The Pure Powers Thesis claims that for any sparse property token, P, (i) P's nature consists entirely of its power and (ii) $\mathrm{P}$ has its power essentially. This implies that P's identity conditions consist of a causal profile - all the possible causal effects that $\mathrm{P}$ can produce - essential to $\mathrm{P}$ being the property it is and metaphysically distinguishing it from other properties. Bird 2007 and Mumford 2004, for instance, posit pure powers in their dispositional essentialist views.

Although the Pure Powers Thesis seems like an alternative formulation of dispositional essentialism, ${ }^{2}$ the view that all properties (including sparse properties) are simultaneously qualitative and powerful also appears consistent with dispositional essentialism, as Jacobs (2011: 81-82) observes. This view originates in the work of C.B. Martin, and is developed by Martin and Heil 1999, Heil 2003, and Martin 2008 without explicitly endorsing dispositional essentialism. Martin and Heil (1999: 47) claim that 'A property just is a certain dispositionality that just is a certain qualitativity.' Heil (2003: 111) maintains that qualities are identical to powers, and Martin (2008: 51) states that the world consists 'of properties that are at once dispositional and qualitative.' So this view asserts the identity of powers and qualities. Following Heil 2010 and Jacobs 2011, I will call such properties powerful qualities. The Powerful Qualities Thesis claims that for any sparse property token, $\mathrm{P}$, (i) $\mathrm{P}$ has a powerful nature, (ii) $\mathrm{P}$ has its powers essentially, (iii) $\mathrm{P}$ has a qualitative nature, and (iv) P's powerful nature $=$ P's qualitative nature. The nature of a property token issues identity conditions specifying what distinguishes one property token from another. The nature of a pure power is de-

\footnotetext{
${ }^{2}$ Specifically, the Pure Powers Thesis may be another way to formulate a strong version of Dispositional Essentialism which maintains that all sparse properties have their powerful natures essentially (a weak version maintains that some but not all sparse properties have their powerful natures essentially).
} 
fined entirely in terms of its causal profile, but for a powerful quality something else is involved.

The nature of powerful qualities will be explored more in section 2. It suffices for now to observe that powerful qualities have a constant manifest nature, making them substantial in a way pure powers are not (Heil 2003: 98, Martin 2008: 32)3. But powerful qualities are not what may be called pure qualities (i.e., categorical properties, which are intrinsically inert). Powerful qualities have the "just-thereness' (Armstrong 2004: 141) of pure qualities or categorical properties combined with the essential powerfulness of pure powers.

Although the Pure Powers Thesis and the Powerful Qualities Thesis understand the internal nature of sparse property tokens differently, both theories posit equally powerful properties with essentially dispositional natures. Thus, they should be viewed as rival ontological accounts of sparse properties within the metaphysics of dispositional essentialism. ${ }^{4}$

The rest of this essay proceeds as follows. In section 2, I discuss the nature of powerful qualities and critique the Powerful Qualities Thesis. I also take notice of a prima facie advantage it has over the Pure Powers Thesis: explaining the being or continuous existence of powers during non-manifesting periods. In section 3 , I identify two levels of objections concerning pure powers, allowing a more exact specification of what it is about the basic nature of pure powers that makes them objectionable to some metaphysicians. In section 4, I formulate and develop the Point Theory of pure powers. This theory provides an explanation of the continuous existence of pure powers when they are latent, thereby neutralizing the most advantageous feature of powerful qualities and securing pure powers as the sparse properties which dispositional essentialists should posit. In developing Point Theory, I examine a problem concerning the relation be-

${ }^{3}$ Heil (2003: 98) says 'If an object's qualities are reduced to or replaced by pure powers, anything resembling substantial nature fades away.'

${ }^{4}$ See Block (forthcoming) for a discussion of the distinction between metaphysics and ontology, which Block applies to philosophy of mind: functionalism is a metaphysics, which can be satisfied by different ontological commitments (e.g., mental properties, physical properties). Similarly, dispositional essentialism is a metaphysics, which can be satisfied by different ontological commitments concerning sparse properties (powerful qualities or pure powers). 
tween powers and space-time points, as well as a problem concerning spatial occupation. I also present an account of the movement of pure powers through space-time.

2 The dense nature of powerful qualities and why it is problematic

To further elucidate the nature of powerful qualities, I will focus on what makes pure powers different from powerful qualities. What does it mean for a property to be qualitative and powerful, not merely powerful? What are the metaphysical consequences of this view?

Assume the indiscernibility of identical objects, $x$ and $y$. So, if $x=$ $y$, then $x$ and $y$ share all the same properties. Now suppose that all the properties $x$ and $y$ share are powerful, and the powers completely and exclusively determine all the possible events $x$ and $y$ are involved in. So, it should make no causal or modal difference whether the powers are powerful qualities or pure powers. Call this No Causal Role Difference. There is no difference in their powers, so $x$ and $y$ will do all the same things in all the same circumstances. Yet the following idea-Qualitative Difference — also seems true: supposing $x$ has pure powers and $y$ has powerful qualities, although No Causal Role Difference is true, $x$ and $y$ cannot be identical. For the quality of $y$ makes it metaphysically if not epistemologically distinguishable from $x$. This clarifies that, because the Powerful Qualities Thesis saturates every causal power with a qualitative nature, the identity conditions of a powerful quality consist of more than just a causal profile. That is, the nature of a powerful quality goes beyond its causal profile.

Martin and Heil (1999: 47) as well Jacobs (2011: 87) use the term 'nature' without explicitly characterizing it. The 'nature' of a property is not a further entity, but the way the property is which yields identity conditions for $\mathrm{P}$ that makes it metaphysically distinct from other property instances. It can provide a way of not only differentiating powerful qualities and pure powers, but differentiating the qualitative from the dispositional within powerful qualities. If it is true of powerful qualities that 'Dispositionality and qualitativity are built into each property; indeed, they are the property' (Martin and Heil 1999: 46), then we can, at least, conceptually distinguish these 
two natures within a unified powerful quality. ${ }^{5}$

It is true that the Powerful Qualities Thesis identifies powers and qualities, so there are not really two natures, since that would imply two entities - properties, sub-properties, aspects, sides, or something else - within a powerful quality. However, powerful qualities do have an ever-manifest, substantial nature: they are ontologically dense compared to pure powers, with more being packed into one property token. The fact that they are dense is suggested by their being more conceptually complex than pure powers. ${ }^{6}$ There should be something ontologically to answer to this conceptual complexity. If it were not logically possible to discern a complexity within the nature of powerful qualities, then there would be no way to distinguish powerful qualities (a more complex nature) from pure powers (a simpler nature). But we can distinguish between them; therefore, we must maintain that powerful qualities have a dense nature (made by their unified dispositionality and qualitativity) that grounds the conceptual complexity associated with them, which we attend to in so distinguishing pure powers from powerful qualities.

Advocates of powerful qualities typically recognize that a conception of sparse properties as pure powers is internally coherent and thus logically possible, although very implausible as contended by Heil 2003 and Jacobs 2011. Therefore, it is clear that the Powerful Qualities Thesis adds something to the nature of sparse propertiesa qualitativity that makes them dense - that is not logically or metaphysically necessary. But it is possible, of course.

So, from the pure powers theorist's perspective, even though

${ }^{5}$ Heil (2003: 173) holds that a property is a way an object is: it is the nature of the object - although we can 'consider' objects and properties separately, they are inseparable. Similarly, we can consider the natures of powerful qualitiestheir qualitative and their powerful natures - separately but they are ontologically inseparable (because identical).

${ }^{6}$ Jacobs (2011: 90) holds that 'To be qualitative is to be identical with a thick quiddity (a quality or a quale)' such that properties differ from each other by their intrinsic nature, not just numerically. The thickness of qualities is similar to what I call their density; however, I want to avoid any phenomenological implications or ties to the term I am using to discuss the nature of powerful qualities, unlike the implications Jacobs (2011: 90-91) makes with the parenthetical inclusion of the term 'quale'. 
powerful qualities are logically and metaphysically possible ${ }^{7}$, they face problems centered around conceptual and ontological simplicity that, ceteris paribus, make pure powers more attractive. This can be seen by applying what I call the causal effects test: if some theoretically posited entity has no possible causal effects, i.e., no causal profile, then it is not spatiotemporally real. ${ }^{8}$ The qualitative nature of $\mathrm{P}$ - whatever $\mathrm{P}$ is that is not pure power - has no possible causal effects other than those given by its powerful nature. One might rejoin that since the qualitative and powerful natures are identical, the quality does have a causal profile. And this is true. Yet, for any sparse property, P, the Powerful Qualities Thesis adds something unnecessary but possible to P's nature, as discussed above: it makes the powerful nature of $\mathrm{P}$ dense by adding qualitativity to it.

Given the causal effects test, now consider this dilemma: either the qualitative nature that is identical to the powerful nature, given by its causal profile, adds something to P's causal profile, or it does not. If it does not, then it does not lead us past the Pure Powers Thesis, for it does not add anything beyond that given by the powerful nature. But if the qualitative nature adds something to the causal profile of $\mathrm{P}$, then it is mysterious what it adds beyond the possible effects issued by the powerful nature. Thus, considerations of simplicity favor pure powers, not powerful qualities.

Both the Powerful Qualities Thesis and the Pure Powers Thesis posit, I presume, the same number of types and tokens of sparse properties within a dispositional essentialist framework. For example, both posit the sparse property charge, and both posit that all the electrons in the universe instantiate charge. All the same existent causal powers of the world are obtained on either view, and thus they are equivalent in terms of their possible manifestations. De-

${ }^{7}$ Suppose powerful qualities are metaphysically possible. What does this mean for dispositional essentialists if, for reasons given in this paper, they accept that sparse properties in the actual world are pure powers? Dispositional essentialists can admit that in some worlds some sparse properties are powerful qualities.

${ }^{8}$ Armstrong (2010: 2) points out that Oddie 1982 formalized the idea that entities we posit should play some causal role, an idea that has its roots in the Eleatic Stranger in Plato's Sophist 247D-E. Armstrong (2010: 2) claims 'if an entity plays no causal role at all, then that is a good argument, though perhaps not a conclusive one, for not postulating that entity.' 
spite this, there remains an unnecessary ontological baggage - an extra bit of being - borne by powerful qualities that pure powers do not carry. Why posit powerful qualities when pure powers afford the same causal possibilities while getting by on something with less density? We should not unless powerful qualities offer some important explanatory advantage.

Because a qualitative nature adds nothing to a property in terms of causal powers, the Powerful Qualities Thesis offers no explanatory benefit over the Pure Powers Thesis regarding the metaphysics of events, processes, or the laws of nature within a dispositional essentialist framework. All these phenomena are explained by reference to causal powers, regardless of the underlying ontology of those causal powers. Nonetheless, it is prima facie plausible that powerful qualities offer one explanatory advantage over pure powers: powerful qualities are better suited as truth-makers for counterfactuals associated with powers, thus explaining the being of sparse properties through latency periods. So, this will be my focus in developing a theory of pure powers.

\section{Two levels of problems for pure powers}

Pure powers face two types of objections. Level 1 objections concern the intrinsic nature of pure powers, including worries about their continuous existence. Level 2 objections concern systems of two or more pure powers, including how substances and qualities can result from a pure powers foundation (Heil 2003: 114-15), how to individuate two or more pure powers with identical causal profiles as Hawthorne 2001 discusses, and the regress argument that nothing would ever get done in a world of pure powers because every power is merely for some manifestation which is simply another power (Martin and Heil 1999: 46). ${ }^{9}$

${ }^{9}$ Martin and Heil (1999: 46) state the regress worry like this: 'Every disposition is a disposition for some manifestation. But if every manifestation is itself purely dispositional, then it will be for some further disposition for some manifestation, and this manifestation, in turn, nothing more than a disposition for some manifestation, ... and so on. A world consisting of pure dispositions would seem to be a world whose inhabitants, although poised to act, never get around to doing anything.' 
There is also a related Level 2 worry that any system of pure powers is completely relational, therefore implausible, because the identity conditions of pure powers must be understood solely in terms of their relation to other powers, as Heil (2003: 97-107, 114) and Jacobs 2011 argue. ${ }^{10,11}$ That is, as Jacobs (2011: 85) observes, the worry for a system of pure powers concerns what enters the various relations that hold between the powers. This ushers in Level 1 worries, but if these worries can be mitigated it could help alleviate some Level 2 concerns because reference can then be made to the intrinsic nature of the relata in a system of pure powers.

Indeed, it seems to me that any system or network of pure powers must have relata: the individual, quality-less, powers themselves. Even though a pure power's identity conditions might be understood in relation to other powers, its being is independent of them. I assume there is a possible world in which only a single pure power, $\mathrm{P}$, is instantiated. P does not necessitate the existence of other powers; thus, genuine Level 1 concerns arise. What does it mean for P, if $\mathrm{P}$ is not a quality, to exist during non-manifestation periods such that counterfactuals associated with $\mathrm{P}$ are true? What, intrinsic to P, admits of P's being? Psillos (2006: 137) asks: If P consists entirely of potential to manifest power - and supposing that any $\mathrm{P}$ need not manifest its power - then what is $\mathrm{P}$ doing when it is not manifesting? Mumford (2006: 481) recognizes this as the 'question of Being' - although he accepts the actuality of pure powers (2006: 485). Ellis (2001: 114) also recognizes the issue, but accepts pure powers while contending that no explanation is needed for their continuous existence.

\section{The point theory of pure powers}

In developing a response to Level 1 concerns about pure powers, I assume the following principle of ontological dependence: for any

\footnotetext{
${ }^{10}$ Holton 1999 argues that a purely relational world is coherent (even if implausible, as powerful qualities theorists hold).

${ }^{11}$ Heil (2003: 114) argues that denial of the Identity Thesis (the Powerful Qualities Thesis, as I have set it up) 'leads to a conception of properties of the fundamental things as pure powers' and this is 'prima facie implausible.'
} 
non-object entities (property tokens, events, units of space-time, etc.), one entity, $E_{1}$, intrinsically ontologically depends on another entity, $\mathrm{E}_{2}$, if and only if $\mathrm{E}_{1}$ cannot exist at $t$ without $\mathrm{E}_{2}$ also existing at $t$, where $\mathrm{E}_{1}$ and $\mathrm{E}_{2}$ are both intrinsic to an object $\mathrm{O}$, or a spacetime location $\mathrm{L}$, such that $\mathrm{O}$ or $\mathrm{L}$ instantiates both $\mathrm{E}_{1}$ and $\mathrm{E}_{2}$. This excludes the possibility that $\mathrm{P}$ is an extrinsic property of $\mathrm{O}$ or $\mathrm{L}$, which would require that $\mathrm{P}$ be grounded in properties of objects or locations other than $\mathrm{O}$ or L, because I aim for an account of P's being that is consistent with its having a wholly intrinsic nature, for reasons given in section 3. The principle permits but does not require grounding in property-less objects or substrata; i.e., although $\mathrm{E}_{1}$ may be grounded in $E_{2}$, where they are both properties of $O, E_{1}$ need not be grounded by $\mathrm{O}$ itself, because property tokens may float free of objects as Schaffer 2003a argues.

The possibility of free-floating properties deserves some explanation. Schaffer (2003a: 125) specifically argues that 'lone properties such as free masses are metaphysically possible - the clustering of properties is merely a contingent fact.' He responds to various objections to this possibility, and then formulates a subtraction principle as the basis of his positive argument for free-floating properties: 'it seems that for any n-propertied object, it is possible for there to be an $\mathrm{n}-1$ propertied subduplicate' (Schaffer 2003a: 136). ${ }^{12}$ That is, for any object, we can keep taking away properties one at a time until we are left with one property: 'no one specific property seems necessary for being an object,' so each is subtractable, all the way down to, for example, free mass (Schaffer 2003a: 136). This implies that, if one is a bundle theorist, there can be no objects with zero properties (Schaffer 2003a: 137). Given that properties might float free, it is not necessary that non-object entities like properties be grounded in objects.

Assuming the above principle of ontological dependence, my proposal is that P's being consists of a causal profile at a space-time point. More precisely, here is my theory of pure powers:

Point Theory: Any pure power token, P, ontologically depends exclusively on (i) the existence of a space-time point, $s$, and (ii)

${ }^{12}$ Schaffer's inspiration for this argument comes from Armstrong (1989: 72). 
and a causal profile at $s$-i.e., $\mathrm{C}_{\mathrm{s}}$ - consisting of a set of fundamental subjunctive facts that make counterfactuals true at $s$.

The idea that subjunctive facts are fundamental comes from Lange 2009 and is discussed in more detail below. Counterfactual statements characterize the subjunctive facts that constitute $\mathrm{C}_{\mathrm{s}}$. These primitive subjunctive facts are the truth-makers, and the counterfactuals are the truth-bearers. $\mathrm{C}_{\mathrm{s}}$ consists of one or more subjunctive facts: one per power, if single-track powers are preferred, or many for each power, if multi-track powers are preferred..$^{13}$ Although the counterfactuals specifying $\mathrm{C}_{\mathrm{s}}$ are true, this does not entail that all truths about P are counterfactual truths. Bostock (2008: 148) observes that 'If powers are entities, there are many truths about the properties objects have (e.g. $a$ has power $P$ ), and these truths are not counterfactual.' If the instantiation of $\mathrm{P}$ does not require an objecte.g., if properties can float free of objects as discussed above - then it will still be true that $\mathrm{P}$ is instantiated at a space-time point, which is not a counterfactual truth.

As mentioned, the proposal that subjunctive facts are fundamental is found in Lange 2009. Lange (2009: 136) 'reverses the standard picture of laws "supporting" counterfactuals.' The standard view (or, at least, one standard view) is that counterfactual truths are true in virtue of laws in conjunction with categorical facts or properties; so if the glass were hit by a hammer, it would shatter is made true by categorical properties of the glass plus laws of nature that govern relations between events. But Lange rejects this account. He argues instead that there are subjunctive facts - characterized by counterfactual truths which have objective truth-values (Lange 2009: 137) — that ground the laws' necessity: 'with these subjunctive facts, we have reached ontological bedrock' (Lange 2009: 136). ${ }^{14}$

${ }^{13}$ Ryle 1949 introduced the idea of multi-track dispositions. For example, if fragility is a multi-track disposition, then an instance of fragility can manifest in multiple ways: cracking, breaking, shattering due to different causes such as a hammer blow, high-pitched singing, etc.

${ }^{14}$ In further detail, Lange (2009: 136) argues (i) that the necessity of the laws is 'what makes them laws' (setting them apart from accidents), (ii) that 'necessity consists of membership in a nonmaximal sub-nomically stable set,' and (iii) therefore that a law 'is a law in virtue of belonging to a nonmaximal sub-nomically 
Notice that dispositional essentialists agree that laws are not fundamental; they argue either that laws supervene on the dispositional essences of things, as in Bird 2007, or that the laws themselves are not ontologically real, as in Mumford 2004. So there is nothing too radical, for the dispositional essentialist, in Lange's proposal regarding laws of nature. But it is Lange's idea of fundamental subjunctive facts that opens up the possibility of Point Theory.

With the core of the Point Theory of pure powers established, I will now examine three aspects of it that are important to understanding its internal plausibility, mitigating Level 1 worries, and establishing the viability of pure powers over powerful qualities. The three aspects addressed below include: the relation between pure powers and space-times points (and thus locations as single points or sets of points), whether pure powers occupy space, and the movement of pure powers through space-time.

\subsection{Pure powers, locations, and space-time points}

The properties typically cited as examples of pure powers by dispositional essentialists are fundamental physical properties, such as mass and charge. A fully complete physics may indicate that some other properties are fundamental: perhaps there will be proto-mass (the power of a particle to gain mass, if the Higgs mechanism in the Standard Model of quantum mechanics is real) and other proto-powers. But space-time points might be pure powers too, and this raises a problem to be discussed below. On Point Theory, what is the nature of the locations (to include either space-time points or sets of spacetime points, i.e., regions) where a causal profile exists?

Locations are either categorical or dispositional properties (assuming they are properties). Ellis (2010: 109) maintains that locations are paradigm examples of categorical sparse properties (many

stable set.' He holds that 'a set of sub-nomic truths is "sub-nomically stable" if and only if whatever the conversational context, the set's members would all still have held under every sub-nomic counterfactual (or subjunctive) supposition that is logically consistent with the set-even under however many such suppositions are nested' (Lange 2009: 29). So, then, the question is what makes true the subjunctives that make the set of laws sub-nomically stable? And the answer is nothing - because the subjunctive facts are fundamental. 
other sparse properties are pure powers on Ellis' view). But a worry for this view is that points or sets of points do not seem to have any qualitative nature to them - it is not clear what their being categorical means. Furthermore, it is problematic if categorical locations ground powers, for this would violate the powers' purity.

If the Pure Powers Thesis and the Point Theory in support of it are correct, then it looks like space-time points must be dispositional. So what are their powers? I suggest that any space-time point will have at least one power (though many will have more, such as fundamental charge and mass) that is pure and fits the requirements of Point Theory: the power of that point to be occupied by some object or further property instance such as mass. ${ }^{15}$ On this view, points are the most fundamental dispositions or powers.

The suggestion that locations possess powers to be occupied raises a worry: if locations are sparse powers, then when they are not manifesting, what are they doing or what does their being consist of ${ }^{16}$ If, on Point Theory, $\mathrm{P}$ consists of a causal profile at a point $s$, then since that location (point) is dispositional and has a causal profile, we would need a new $s$, thus generating an infinite series of points, along with an infinite series of causal profiles, necessary for P. I offer two possible responses to this objection that avoid positing pure qualities, categorical properties (if these are different from pure qualities), or powerful qualities.

First, to take the objection head on, an infinite series of points with corresponding causal profiles might be implausible, but it is not incoherent. And, it may not be as implausible as it first seems. There is reason to believe it is possible that reality consists of an infinite number of levels. For example, Schaffer (2003b: 505-506) claims there is no evidence for a fundamental level of reality. Reality might have a fundamental level or an 'infinite descent' of levels, but the 'empirical evidence to date is neutral as to which structure science

${ }^{15}$ If Point Theory is correct, and if we are to maintain the possibility of a one-power world, then there is a possible world with only one space-time point (it would be a point with a single pure power token). This is because for any additional point that exists, it would necessarily have some power (to be occupied), thus negating the possibility of a one-power world.

${ }^{16}$ Thanks to an anonymous reviewer for raising this objection. 
is reflecting. And so, concerning the proposition that there exists a fundamental level of nature, one should withhold belief' (Schaffer 2003b: 505-506)..$^{17}$ If this correct, then we should remain open to the possibility that there are infinite sub-locations - locations within locations, finer and finer space-time points, or points in descending sub-spaces - corresponding to each new sub-level of reality. If this is on the right track, then when power $\mathrm{P}_{1}$ at a higher level is not manifesting, its being resides in $\mathrm{C}_{\mathrm{s}}$ at point $s_{1}$, but there is a power $\mathrm{P}_{2}$ (necessary and sufficient for $\mathrm{P}_{1}$ ) that is manifesting for $\mathrm{P}_{1}$, and $\mathrm{P}_{2}^{2}$ 's being resides in a corresponding $\mathrm{C}_{\mathrm{s}}$ at sub-point $s_{2}$, and so on. So there could be infinitely descending levels of power-some extra power does not hurt one's ontology. On this view, then, what is a pure power doing when not manifesting? Well, it is grounded in some further pure power - a causal profile at a point - that is manifesting, and so on.

Second, if one wants to do without infinite levels, it is possible that the point where $\mathrm{P}$ is instantiated just is identical to a causal profile $\mathrm{C}_{\mathrm{s}}$ (which in turn constitutes the power to be occupied). However, this would imply that Point Theory is not fully comprehensive for sparse pure powers (since Point Theory postulates points and causal profiles as the basis of all pure powers); but, it does fit or account for sparse, non-point pure powers, even those powers like mass and charge whose causal profiles exist at space-time points that are themselves pure powers.

It might be contended that since the subjunctive facts that constitute $\mathrm{C}_{\mathrm{s}}$ are fundamental, they do not ontologically need space-time points. Lange 2009 does not appear to require a connection between space-time points and fundamental subjunctive facts (although he is not primarily concerned with sparse properties, but with subjunctive facts grounding the laws of nature). Point Theory does not deny that subjunctive facts are fundamental, it just says that they are necessarily tied to space-time points as a spatiotemporal condition, or anchor, of their reality; points and subjunctive facts are co-fundamental, and this combination makes for pure powers (unless the second response to the worry is deemed more plausible, since then there is no com-

${ }^{17}$ Also see Dehmelt 1989, who postulates infinite sub-electronic levels of structure. 
bination, there is just the point that is identical to the causal profile).

\subsection{Pure powers and spatial occupation}

Point Theory implies that $\mathrm{P}$ is an actual, continuously instantiated property, consistent with other views about pure powers, e.g., Mumford (2006: 485) and Bostock (2008: 145). Martin (2008: 32) claims that the 'readinesses' of dispositions 'are all actual' and I agree, without adding as he does that this readiness needs qualitativity. Just as with a powerful quality, $\mathrm{P}$ is an actual property - ready to manifest - while latent. But P's actuality requires a spatiotemporal condition of some sort. Point Theory provides that, but denies the claim that $\mathrm{P}$ requires spatial occupation as a quality might require.

I assume a distinction between being instantiated at a point in space versus being instantiated in a region of two or more points (thus occupying space by creating an extension or distance). P can be instantiated at a point which is not extended - per Point Theory — and thus does not occupy space in that sense. Consider a world with infinite spacetime but no extended objects: space is empty or non-occupied. Yet infinite counterfactuals might be true of a single point in space, and through infinite levels if the first response to the objection in section 4.1 is correct, yet nothing occupies space.

This is important because a possible objection is that if $\mathrm{P}$ is instantiated, then during non-manifestation periods $\mathrm{P}$ should occupy a spatial region $R$, where $R$ consists of a set of two or more simultaneous points immediately neighboring each other. ${ }^{18}$ If this conditional is true, the problem for pure powers seems to be that there is nothing to occupy $R$ in the way that an object or a structural property token (like shape) occupies space; thus, P ceases to exist when latent. This worry about spatial occupation is a manifestation of worries about P's continuous existence when latent. But if Point Theory is accurate, then the spatial occupation objection is a pseudo-problem because $\mathrm{P}$ can be instantiated at a point, and a point with a causal profile does not need to be extended in, or occupy, space. Spatial occupation is, perhaps, a condition for powerful qualities or pure

${ }^{18} \mathrm{I}$ assume that during manifesting periods, $\mathrm{P}$ might present itself qualitatively or be involved in some spatially occupying event. 
qualities (i.e., categorical properties), but not for pure powers.

Suppose, however, it is true that if a stimulus $\mathrm{S}$ occurs in $R$, then manifestation $\mathrm{M}$ will occur. Thus, it appears $\mathrm{P}$ is spatially extended because it is instantiated throughout $R$. However, at any point in $R$, all the counterfactuals specifying $\mathrm{C}_{\mathrm{s}}$ will be true because $\mathrm{C}_{\mathrm{s}}$ holds at every point $s$ in $R$. Therefore, what appears to be a single pure power spread throughout $R$ consists of a set of many tokens of pure powers instantiated at all the points in $R$.

Williams (2009: 17-18) objects to pure powers based on concerns about spatial occupation. But he is mainly interested in showing that if a sub-atomic particle $x$ has pure powers, then $x$ must still be some 'way' at all times, which involves spatial occupation and requires some categoricalness (or, qualitativity); therefore, since purportedly pure powers appear to be grounded in categoricalness, they are not really pure. Precedent for this type of worry is found, for instance, in Blackburn 1990. But these specific concerns are somewhat tangential to my discussion, since I'm assuming that any pure power, $\mathrm{P}$, can float free of and thus exist (i.e., remain instantiated) independently of objects, as discussed at the beginning of section 4 in formulating a principle of ontological dependence; therefore P's occupation of space, via its object bearer in some way, is not necessary. Besides, subatomic particles (objects) might be point particles which instantiate pure powers.

Molnar (2003: 133-134), in defending pure powers, contends that fundamental particles are simple and completely lacking structure, which suggests the possibility of point particles (to be physically extended is to have some structure). So, assuming point particles count as objects and pure powers can be properties of point particles, if $\mathrm{P}$ is borne by an object this does not necessitate P's occupying space. That would only be true if particles necessarily occupied space. Therefore, on Point Theory, the instantiation of P by a particle can avoid any of the qualitative or categorical nature that arguably comes along with spatial occupation.

\subsection{The movement of pure powers through space-time}

Although space-time points do not move, $\mathrm{P}$ can move or shift between points, thus accounting for the movement of pure powers, and 
the objects bearing them, through space-time. This occurs either (i) as P's bearer moves (e.g., a particle with mass moving through space-time), or (ii) if property tokens can 'float free' of their bearers as Schaffer 2003a argues, then as P itself moves. On either option, as $\mathrm{P}$ moves between points, $\mathrm{C}_{\mathrm{s}}$ shifts between those points. Causal processes involving multiple powers can be accounted for this way, by a series of shifts in the location of $\mathrm{C}_{\mathrm{s}}$.

These contentions can be developed along lines consistent with one of the axiomatic systems for topology and physics developed by Carnap 1958. ${ }^{19}$ An extensive development of this is worthwhile, but my modest aim here is to point out the basic features most relevant to Point Theory.

Carnap (1958: 197) uses a logic of relations 'to treat topological properties of space and time by a purely topological method' and thus with no use of concepts with a 'metric (non-topological) character.' He provides three distinct logical systems for describing the nature of world-points within the framework of Einstein's general theory of relativity. One of these systems, the Wlin-System (Carnap 1958: 207-9), appears tailor-made for the conception of pure powers given by Point Theory. In the Wlin-System, 'world-points are again [as with Carnap's C-T System (1958: 197-207)] taken as individuals - however, world-points not as particle slices [as with the C-T System], but as the space-time points corresponding thereto' (1958: 207). That is, the world-points that make up a world-line of an individual (e.g., a particle) just are space-time points. The world-line of a particle consists of a class of time relations (Carnap 1958: 207) that specify the temporal moments of the particle along its path. Coincident world-points are identical (Carnap 1958: 207).

If world-points are space-time points per Carnap's Wlin-System, and these are fundamental individuals or 'particles', then they should possess some fundamental or sparse properties. If these sparse properties are pure powers, then it follows that they are pure powers of world-points. That is, particles bearing pure powers just are spacetime points (the world-points) on the Wlin-System, consistent with Point Theory. These particles need not be substrata existing independently of their properties, for they might be just bundles of pow-

${ }^{19}$ Thanks to Gary Merrill for suggesting this. 
er tokens existing at space-time points; alternatively, perhaps the individuals or world-points are simply property tokens.

On the Wlin-System, as with the other axiomatic systems Carnap develops for physics, a signal relation holds between points: 'An effect reaches from a world-point $x$ to a world-point $y$ if and only if $x$ is connected to $y$ by a signal' (Carnap 1958: 201). A signal can occur between a single individual (a world-point on the Wlin-System) at an earlier time and a later time on its world-line, or a signal can occur between distinct world-points, thus linking 'particles' or individuals by linking their world lines, as when one particle's momentum or energy is transferred to another. The signal relation is comparable to the relation of a power to its manifestation. So, the relevance of this to P, on Point Theory, is this: in the case of a single world-point, $s$, the status (as defined by $\mathrm{C}_{\mathrm{s}}$ ) of $s$ at $t_{1}$ affects its later status at $t_{2}$ (i.e., this is just a relation between spatiotemporal stages of $\mathrm{P}$ ). In the case of signals between two world-points, $s^{1}$ and $s^{2}, s^{2}$ may receive the stimulus from $s^{1}$ and thus manifest, connecting the two world-points by the manifestation relation (by analogy, consider one billiard ball striking another, causing it to accelerate, thus a signal relation obtains).

If these contentions are correct, the Wlin-System provides an axiomatic system for fundamental physics that is consistent with, and bolsters, Point Theory. P's movement is a shifting of $\mathrm{C}_{\mathrm{s}}$ between points along a world-line. Since $\mathrm{C}_{\mathrm{s}}$ holds consecutively along the points of a particular world-line, this accounts for P's identity along its world-line.

\subsection{Concluding remarks}

As a theory of pure powers, Point Theory does without mysterious qualities, powerless natures that are somehow identical to powerful natures. Point Theory maintains that sparse properties are simply, purely, powerful: they consist of causal profiles at space-time points.

For any given pure power, when it is not manifesting, it exists or is instantiated qua power because there remains an actual causal profile - a set of fundamental subjunctive facts - and an actual space-time point that stands in being. These space-time points might themselves be powers, perhaps with an infinite series of grounding 
causal profiles at sub-points (as discussed in section 4.1). On Point Theory, pure powers need not occupy space in the sense of being extended like a quality is expected to be (as discussed in section 4.2), and they can move (or shift) through space-time per the mechanisms detailed in Carnap's Wlin-System (as discussed in section 4.3).

How do qualities come to be, at non-sparse levels of reality, out of pure powers? That is a Level 2 issue. But notice that unless we contend that all properties at all levels are pure powers, nothing prevents us from maintaining that some manifestations of some pure powers are qualities or have a qualitative nature.

Based on these considerations, I conclude that Point Theory mitigates Level 1 worries about pure powers, effectively neutralizing the explanatory advantage that the Powerful Qualities Thesis appeared to have over the Pure Powers Thesis. Therefore, dispositional essentialists should posit pure powers. ${ }^{20}$

William A. Bauer Department of Philosophy and Religious Studies North Carolina State University 340 Withers Hall, Campus Box 8103 Raleigh, NC 27695-8103, USA wabauer@ncsu.edu

\section{References}

Armstrong, David. 1989. Universals: An Opinionated Introduction. Boulder: Westview Press.

Armstrong, David. 2004. Truth and Truthmakers. Cambridge: Cambridge University Press.

Armstrong, David. 2010. Sketch for a Systematic Metaphysics. Oxford: Oxford University Press.

Bird, Alexander. 2007. Nature's Metaphysics. Oxford: Oxford University Press.

Blackburn, Simon. 1990. Filling in Space. Analysis, 50: 62-65.

Block, Ned. Forthcoming. Functional Reduction. In Supervenience in Mind: A Festschrift for Jaegwon Kim. Edited by T. Horgan, D. Sosa, and M. Sabates. Cambridge: MIT Press.

Bostock, Simon. 2008. In Defence of Pan-Dispositionalism. Metaphysica, 9(2):

${ }^{20}$ I would like to thank John Carroll, Eric Carter, Gary Merrill, Michael Pendlebury, Stephen Puryear, and Jessica Wilson for valuable discussion. Thanks also to audience members at the Society for Exact Philosophy meeting at the University of Manitoba on May 27, 2011, and audience members at the Joint Meeting of the North Carolina Philosophical Society and the South Carolina Society for Philosophy at Elon University on February 25, 2012. 
139-157.

Carnap, Rudolf. 1958. Introduction to Symbolic Logic and Its Applications. New York: Dover.

Dehmelt, Hans. 1989. Triton,...Electron,..., Cosmon...: An Infinite Regression? Proceedings of the National Academy of Sciences, 86: 8618-8619.

Ellis, Brian. 2001. Scientific Essentialism. Cambridge: Cambridge University Press.

Ellis, Brian. 2010. The Metaphysics of Scientific Realism. Montreal: McGill-Queen's University Press.

Hawthorne, John. 2001. Causal Structuralism. Philosophical Perspectives, 15: 361 378

Heil, John. 2003. From an Ontological Point of View. Oxford: Oxford University Press.

Heil, John. 2010. Powerful Qualities. In The Metaphysics of Powers: Their Grounding and their Manifestations. Edited by Anna Marmodoro. New York: Routledge.

Holton, Richard. 1999. Dispositions All the Way Round. Analysis, 59: 9-14.

Jacobs, Jonathan. 2011. Powerful Qualities, not Pure Powers. The Monist, 94(1): 81-102

Lange, Marc. 2009. Laws and Lawmakers: Science, Metaphysics, and the Laws of Nature. Oxford: Oxford University Press.

Lewis, David. 1986a. Introduction. Philosophical Papers, vol. II. Oxford: Oxford University Press.

Lewis, David. 1986b. On the Plurality of Worlds. Oxford: Basil Blackwell.

Martin, Charles Burton. 2008. The Mind in Nature. Oxford: Oxford University Press.

Martin, Charles Burton \& John Heil. 1999. The Ontological Turn. Midwest Studies in Philosophy, 23: 34-60.

Molnar, George. 2003. Powers: A Study in Metaphysics. Oxford: Oxford University Press.

Mumford, Stephen. 2004. Laws in Nature. New York: Routledge.

Mumford, Stephen. 2006. The Ungrounded Argument. Synthese, 149: 471-489.

Oddie, Graham. 1982. Armstrong on the Eleatic Principle and Abstract Entities. Philosophical Studies, 41(2): 285-295.

Plato. Sophist. Translated by Nicholas P. White. In Plato: Complete Works. Edited by J. M. Cooper and D.S. Hutchinson. Indianapolis: Hackett, 1997.

Psillos, Stathis. 2006. What do powers do when they are not manifested? Philosophy and Phenomenological Research, 72(1): 137-156.

Ryle, Gilbert. 1949. The Concept of Mind. Chicago: University of Chicago Press.

Schaffer, Jonathan. 2003a. The Problem of Free Mass: Must properties cluster? Philosophy and Phenomenological Research, 66(1): 125-138.

Schaffer, Jonathan. 2003b. Is there a fundamental level? Noûs, 37(3): 498-517.

Williams, Neil. 2009. The Ungrounded Argument is Unfounded: A Response to Mumford. Synthese, 170(1): 7-19. 